\title{
Modeling and Simulation of Energy Management System for Smart City with the Formalism DEVS: Towards Reducing the Energy Consumption
}

\author{
Abdelfettah Maatoug \\ National superior School of \\ Computer Science, Algiers \\ Algeria
}

\author{
Ghalem Belalem \\ Department Of Computer Science \\ University Of Oran \\ Algeria
}

\author{
Kadda Mostefaoui \\ Department Of Computer Science \\ University Of Tiaret \\ Algeria
}

\begin{abstract}
A simulation of a dynamic ecosystem such as the smart city can test new concepts for reducing energy consumption. Research related to energy management can be divided into two categories: predictive control (anticipative) and adaptive control (reactive). A new energy management system of a building (BEMS) which is the chosen system to validate treats a long-term anticipative control and introduces a reactive control that adds another level of intelligence to the BEMS. This work is focused on modeling and simulation of this system using the formalism (DEVS). Our motivation is explained by the fact that DEVS is a tool for modeling of discrete event systems and it decomposes the overall system into subsystems in order to facilitate the achievement which is consistent with the characteristics of multilayer architecture of the chosen system.
\end{abstract}

\section{General Terms}

Energy Management System, Energy management, Energy consumption, Atomic model, Discrete event system, Reactive layer, Coupled model, Predictive control, Local layer, Anticipative layer, Multisource and Multiload building, Multilevel model.

\section{Keywords}

Smart city, a Building Energy Management System (BEMS), Reduction of energy consumption, Multilayer Architecture, Modeling and Simulation, The formalism DEVS.

\section{INTRODUCTION}

In the future smart city, new technologies of information and communication will allow better management of available resources. The future infrastructure of energy system appears as a complex system in which the improved monitoring and control of energy generation and / or consuming entities in the system is possible.

In this system, these entities will no longer be considered as black boxes but interconnected to provide information regarding their energy behavior. This will result in several approaches that will boost energy efficiency.

A simulation of a dynamic ecosystem such as the smart city will enable us to test new concepts for reducing energy consumption. Thus, for reasons of cost and time, an Energy Management System (EMS) of a smart city cannot be designed specifically for each building, so we will look at a Building EMS (BEMS).

This work is focused on modeling and simulation of a BEMS using the characteristics of the formalism (DEVS). Our motivation is explained by the fact that DEVS is a tool for modeling of discrete event systems and it decomposes the overall system into subsystems in order to facilitate the achievement which is consistent with the characteristics of multilayer architecture of the chosen system.

This paper is organized as follows: In the section 2 we quote some energy managers. In the section 3 we introduce the basic concepts of DEVS, we propose a multilevel model for the energy management in multisource and multiload building and we discuss the results of the model simulation. Finally we conclude our work in the section 4 and we give our future prospects.

\section{STATE OF THE ART}

Several researches led to the development of energy management systems in buildings.

\subsection{Energy Managers}

Before giving the architecture and mechanism of the selected BEMS, we will quote some energy managers:

G. J. Levermore and D. Kolokotsa presented related works to energy management and cost strategies and forecasting energy consumption [1] [2], while F. Calvino and N. Morel focused on fuzzy logic, neural networks and the optimal or predictive control of thermal conditions in homes [3] [4]. In [5], analyses of management techniques of the load have been detailed.

According to K. Wacks, a BEMS contains methods that coordinate the activities of consumers and energy providers to better adapt the capacity of energy production with the needs of consumers to avoid peaks of energy demand and their negative effects [6].

Over the past three years, most researches focus on the management and control of loads by the technologies of «Smart Grid». These technologies can reduce domestic energy consumption (electricity and heating) and optimize the import / export of electricity through smoothing of the load curve [7].

All this research can be divided into two categories: predictive control (anticipative) and adaptive control (reactive: realtime). Most recent developments on BEMS use the concept of predictive control. This control uses a model with measured data to predict the optimal control strategy to implement [8]. The predictive control is used, an error of forecasting in shortterm (10-20 $\mathrm{min})$ or long term (days) for the control of temperature or humidity. Both are within acceptable limits for the control of temperature and humidity.

The second category of works also uses predictive control, but introduces control algorithms in real time. 
G. P. Henze offered an optimal adaptive control for autonomous hybrid PV system with storage [9].

These projects correspond to different cases of applications, but they have a common objective to the establishment of an energy management system in a building in order to optimize the use of energy especially in the case of multisource and multiload buildings [10] (Figure 1):

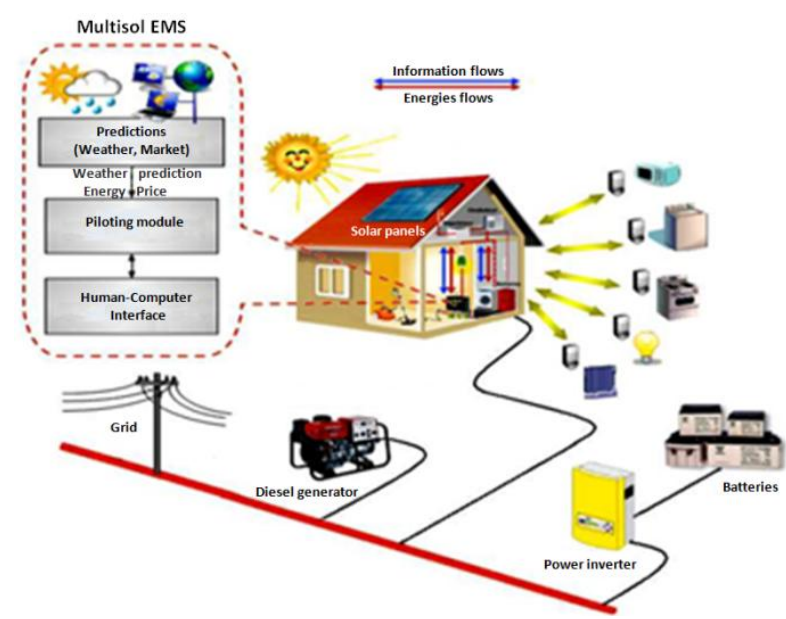

Fig 1: Energy management in a multisource and multiload building [11]

A. Shadi proposed a Multi-agent Distributed System which defines two levels of BEMS control at different horizons of time: the Reactive mechanism and the Anticipative mechanism.

D. L. Ha offered a management system with anticipative and reactive control of the building management, as is the case of the BEMS to validate, that treats a anticipative long-term control (days) and introduces the reactive control that adds another level of intelligence to the BEMS. The architecture and the function of the management system chosen will be detailed later.

\subsection{The multilayer and multiscale control system}

Several levels of modeling are used in this architecture. They correspond to several layers of command to divide the overall problem into sub- problems to be more responsive to uncertainties. Between these layers, a flow of information is used to exchange instructions and emergency messages. These levels of management correspond to prioritized algorithms for solving according to different time scales.

An optimization solution is calculated at the highest level, that is to say, with the longer sampling period, taking into account the relatively inaccurate predictions. The solution obtained is then refined in a lower level of management that fits the solution already calculated taking into account more detailed information for a smaller sampling period. The solution should converge towards real instructions to equipments in the building, or towards advices to occupants.

This architecture of multi- levels management must address to the major problem in the management of energy flows: the prevailing uncertainties in the model of behavior of services in the external conditions and especially in the behavior of forecasts and demands of people. Management architecture in three layers proposed by D. L. Ha: an anticipative layer, a reactive layer and a local layer is shown in the following figure:

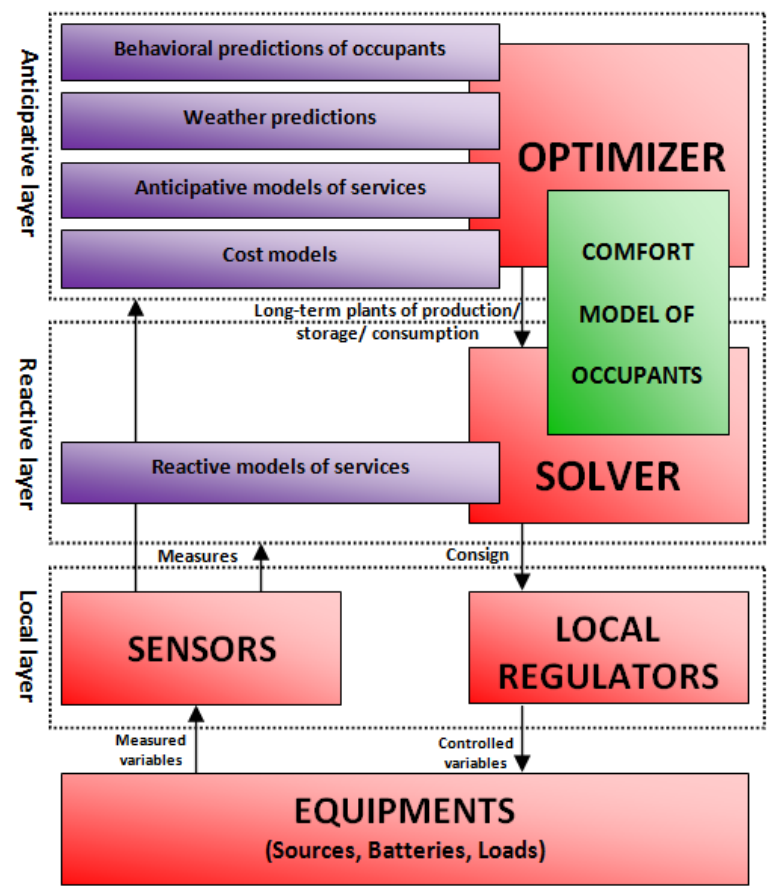

Fig 2: Multilayer mechanism of piloting for energy management in the habitat [12]

\subsubsection{The anticipative layer}

The anticipative layer aims to plan consumption and production of energy when events are expected few hours or a day in advance. The control principle of this layer is based on weather forecasts, schedules of services by occupants and predicting their presence, the electricity market and power available (subscription ). Anticipated solutions calculated by this layer are recalculated when new forecasts are available.

\subsubsection{The reactive layer}

The reactive layer helps the anticipative layer to realize the allocation plan of energy resources, taking into account energy constraints and comfort of the user in real time. If the energy source is unavailable or restricted, the reactive layer will involve stopping the consumption of certain services (to satisfy). As against, if there is no unexpected event, this layer does not intervene. In this case, its role is to transmit instructions to the local layer.

\subsubsection{The local layer}

The local layer allows applying the instructions that comes from the reactive layer. For example, in the thermal environments, this layer functions in all or nothing to maintain the temperature of a thermal environment to near values of the set temperature calculated by the anticipative layer then adjusted by the reactive layer [13] .

This work is focused on the first two layers of control because the local layer is a matter for equipment manufacturers. Our goal is not only to model these control layers but also to validate their operation and the entire energy management mechanism by varying scenarios predicted (from BEMS) and real data (simulation values from the simulation model of building). 


\section{MODELING AND SIMULATION}

In general, the main objective of validation is to check if a system works as expected, in different conditions and scenarios of simulation. The validation aims to make the model useful in the sense that it responds to good problem.

\subsection{Modeling and discrete event simulation (DEVS)}

The formalism DEVS (Discrete Event System Specification) is a modeling approach based on general systems theory. It is a modular and hierarchical formalism for modeling, centered on the concept of state. The constructed models are described with discrete event formalisms [14].

The formalism DEVS defines two categories of models: atomic models and coupled models.

\subsubsection{The atomic model}

Atomic models are loaded to represent the system behavior. DEVS considers an atomic model as a mathematical concept based on time, a set of values characterizing all possible stimuli in inputs and outputs of the system and two functions to determine the behavioral response to these stimuli (Figure 3) [15].

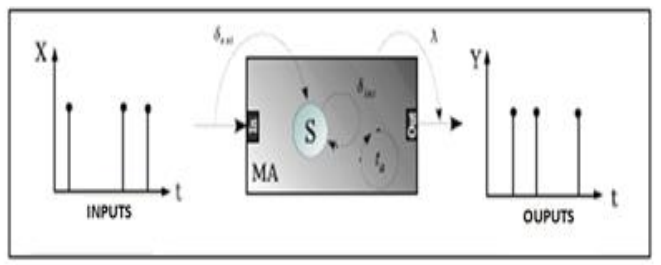

Fig 3: Atomic model in action

An atomic model is specified by:

$$
M A=\left(\mathrm{X}, \mathrm{Y}, \mathrm{S}, \delta_{\text {ext }}, \delta_{\text {int }}, \lambda, \mathrm{ta}\right)
$$

where

$\mathbf{X}$ : set of input events.

Y: set of output events.

$\mathbf{S}$ : set of possibly infinite states.

$\boldsymbol{\delta}_{\text {int }}$ : internal transition function caused by the occurrence of internal events.

$\boldsymbol{\delta}_{\text {ext }}$ : external transition function caused by the occurrence of external events.

$\lambda$ : output function.

ta: a state function lifetime ( $\mathrm{t}_{\max }$ of a state model $\left.\mathrm{s} \in \mathrm{S}\right)$.

\subsubsection{The coupled model}

The formalism DEVS uses the notion of the hierarchy of description which allows the construction of models called «coupled» from a set of sub- models and three coupling relationships with these sub- models [15].

Coupled model consists of sub- models that can be atomic or coupled and has three coupling relationships: internal coupling relationships, external inputs and external outputs relationships (Figure 4):

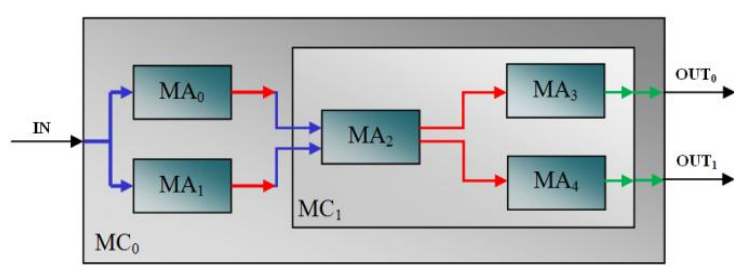

Fig 4: Hierarchy of DEVS modeling

The coupled model of discrete event is defined by: $M C=<\mathrm{X}, \mathrm{Y}, \mathrm{D},\left\{\mathrm{M}_{\mathrm{d}} / \mathrm{d} \in \mathrm{D}\right\}, \mathrm{EIC}, \mathrm{EOC}, \mathrm{IC}>\quad$ where

$\mathbf{X}$ : set of external events.

Y : set of output events .

D : set of component names .

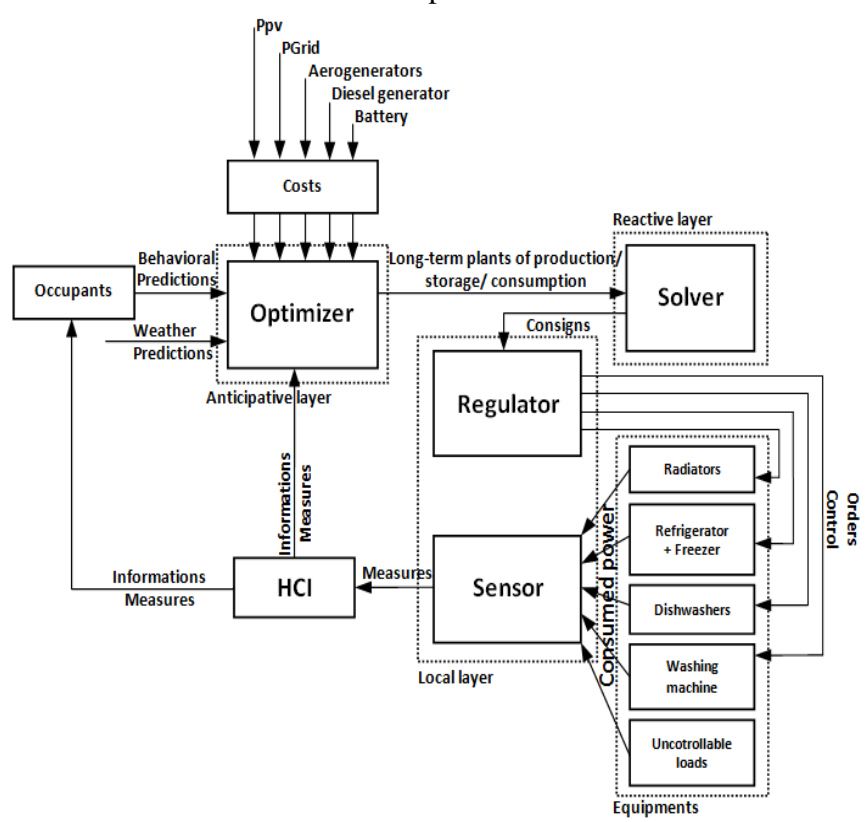

Fig 5: Multilevel model for the energy management in multisource and multiload building

$\mathbf{M}_{\mathbf{d}}$ : DEVS model.

EIC: external input couplings.

EOC : External output couplings .

IC: internal couplings.

\subsection{Modeling of the BEMS with DEVS}

We have designed the model below for multisource and multiload building respecting the multi-levels architecture which has been described previously:

\subsubsection{The formalization of the proposed model in} DEVS

The model we propose divides the BEMS system into eight subsystems: Costs, Optimizer, Solver, Regulator, Equipments, Sensor, HCI and Occupants.

We give to a BEMS the following structure of a DEVS coupled model:

$$
\begin{gathered}
B E M S=<\mathrm{X}, \mathrm{Y}, \mathrm{D},\left\{\mathrm{M}_{\mathrm{d}} / \mathrm{d} \in \mathrm{D}\right\}, \mathrm{EIC}, \mathrm{EOC}, \mathrm{IC}>\text { Where } \\
X=\left\{(p, v) / p \in \text { IPorts }, v \in X_{p}\right\} \quad \begin{array}{r}
\text { Inputs values } \\
\text { they can take. }
\end{array}
\end{gathered}
$$




$$
Y=\left\{(p, v) / p \in \text { OPorts }, v \in Y_{p}\right\} \quad \begin{gathered}
\text { Outputs values } \\
\text { they can take. }
\end{gathered}
$$

D Set of names of the atomic or coupled models that compose the BEMS: $\mathrm{D}=\{$ Costs, Optimizer, Solver, Regulator, Equipments, Sensor, HCI, Occupants \}

$\mathbf{M}_{\mathbf{D}}$ is the set of atomic or coupled the models that compose the BEMS:

$\mathrm{M}_{\text {Costs }}$ is the model of costs subsystem.

$\mathrm{M}_{\text {Optimizer }}$ is the model of Optimizer subsystem....and so on.

$$
E I C=\left\{((B E M S, a),(d, b)) / a \in \text { IPorts }_{s}, b \in \text { IPorts }_{d}\right\}
$$$$
E O C=\left\{((d, b),(B E M S, a)) / a \in \text { OPorts }_{B E M S}, b \in \text { OPorts }_{d}\right\}
$$$$
I C=\left\{((i, a),(j, b)) / i, j \in D, i \neq j, a \in \text { OPorts }_{i}, b \in \text { IPorts }_{j}\right\}
$$

EIC defines ports of BEMS model that are connected to ports of components models that receive external events. EOC defines ports of BEMS model that are connected to ports of components models that emit events.

\subsubsection{Representation of the proposed model using the JDEVS tool}

Figure 6 shows the proposed model BEMS modeled on JDEVS environment. This model consists of several atomic models:

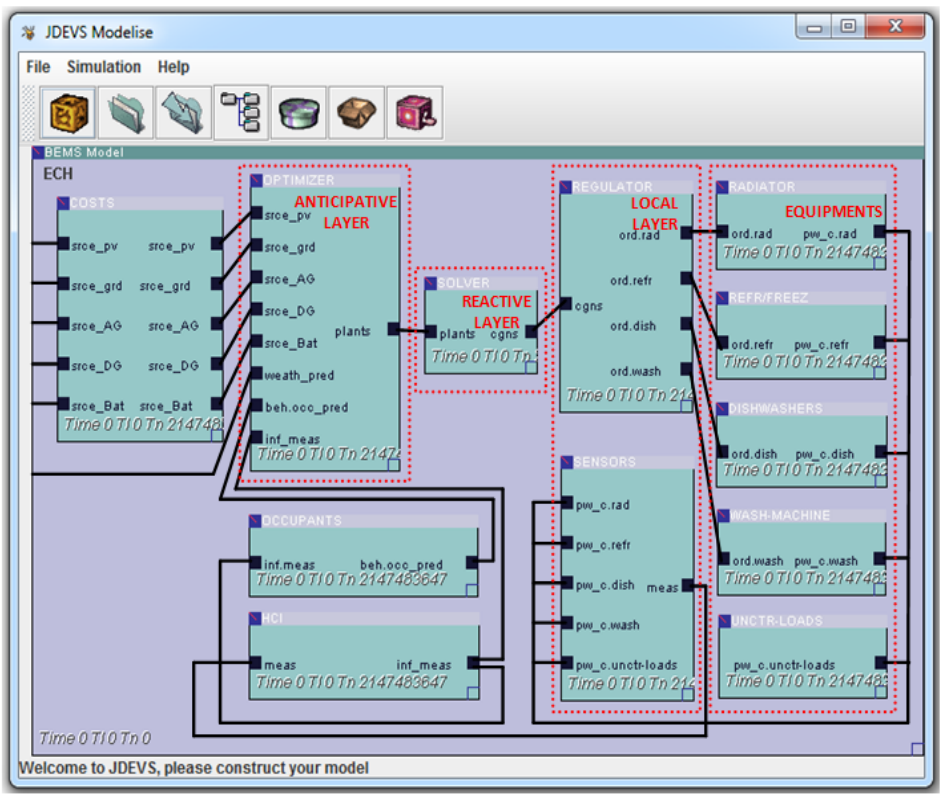

Fig 6: Representation of the BEMS model with JDEVS

\subsubsection{Simulation of a thermal model: a case of application}

Cases of applications include: PV sources, RE, and the following loads:
Table 1. Available loads in the building

\begin{tabular}{|c|c|c|c|c|}
\hline Equipments & $\begin{array}{c}\text { Power } \\
(\mathbf{W})\end{array}$ & Number & Command & $\begin{array}{c}\text { Interrup } \\
\text {-tible }\end{array}$ \\
\hline Radiator & 1500 & 1 & $\mathrm{~T}_{\text {cgn/ON_OFF }}^{\circ}$ & Yes \\
\hline Refrigerator & 150 & 1 & $\mathrm{~T}_{\text {cgn/ON_OFF }}^{\circ}$ & Yes \\
\hline Freezer & 200 & 1 & $\mathrm{~T}_{\text {cgn/ON_OFF }}^{\circ}$ & Yes \\
\hline $\begin{array}{c}\text { Washing } \\
\text { machine }\end{array}$ & 2000 & 1 & $\mathrm{t}_{\text {of START }}$ & Not \\
\hline $\begin{array}{c}\text { Dishwasher } \\
\text { uncontrollable } \\
\text { loads }\end{array}$ & 2200 & 1 & $\mathrm{t}_{\text {of Start }}$ & Not \\
\hline
\end{tabular}

We are interested in the energy behavior of Radiator without and with the BEMS to test the operation of the system.

\subsubsection{The Radiator sub-model}

The Radiator is a thermal service as it is regulated as follows: it is either in the stop $(\mathrm{P}=0 \mathrm{~W})$ or in the maximum power $\left(\mathrm{P}=\mathrm{P}_{\max }\right)$. So $\mathrm{t}_{\max }$ is the period of operation at $\mathrm{P}_{\max }$ and $\mathrm{t}_{\min }$ the period of stop, the model is [16]:

\subsection{At the period «t $\boldsymbol{t}_{\text {max }}$ "}

$$
\mathrm{d}\left(\mathrm{T}_{\text {in }}-\mathrm{T}_{\text {out }}\right) / \mathrm{dt}=1 / \tau\left(\mathrm{T}_{\text {in }}-\mathrm{T}_{\text {out }}\right)+\mathrm{k} / \tau\left(\mathrm{P}_{\text {max }}\right)
$$

$\mathbf{T}_{\mathbf{f}}$ : The temperature provided by the Radiator $\left[{ }^{\circ} \mathrm{K}\right]$ $\mathbf{T}_{\text {out }}$ : The outside temperature ( room temperature ) $\left[{ }^{\circ} \mathrm{K}\right]$ k: A factor of heat generation for Radiator ( intrinsic characteristic )

$\tau$ : time constant of Radiator [s] such that $\tau=\mathrm{R} \times \mathrm{C}$ with "R" in $[\mathrm{K} / \mathrm{W}]$ is the thermal resistance and " $\mathrm{C} "$ in $[\mathrm{J} / \mathrm{K}]$ is the calorific capacity of the Radiator. We took an average constant of time.

$\mathbf{P}_{\text {max }}$ : maximum power of Radiator [W]

By integrating (1) we get:

$\mathrm{T}_{\mathrm{rd}}+\Delta \mathrm{T}-\mathrm{T}_{\text {out }}=\mathrm{e}^{-t_{\max } / \tau}\left(\mathrm{T}_{\mathrm{rd}}-\Delta \mathrm{T}-\mathrm{T}_{\text {out }}\right)+\mathrm{k}\left(1-\mathrm{e}^{-t_{\max } / \tau}\right) \mathrm{P}_{\max }$

$\mathbf{T}_{\mathbf{r d}}:$ Radiator temperature of consign

$\Delta \mathbf{T}$ : Variation of maximum temperature allowed for the command (here $=1{ }^{\circ} \mathrm{K}$ )

\subsection{At the period « $\boldsymbol{t}_{\boldsymbol{m i n}}$ "}

$\mathrm{d}\left(\mathrm{T}_{\text {in }}-\mathrm{T}_{\text {out }}\right) / \mathrm{dt}=1 / \tau\left(\mathrm{T}_{\text {in }}-\mathrm{T}_{\text {out }}\right)$

By integrating (3) we get:

$\mathrm{T}_{\mathrm{rd}}-\Delta \mathrm{T}-\mathrm{T}_{\text {out }}=\mathrm{e}^{-t_{\min } / \tau}\left(\mathrm{T}_{\mathrm{rd}}+\Delta \mathrm{T}-\mathrm{T}_{\text {out }}\right)$

The energy management system calculates the temperature of consign and imposes it on the system. The model provided the periods of operation $t_{\min }$ and $t_{\max }$ as a function of the set point temperature of the room in which the Radiator located, and the intrinsic parameters of the Radiator, and power consumption based on cost. 


\subsubsection{Formalization of the Radiator subsystem in DEVS}

We formalize the Radiator subsystem by the structure of an atomic DEVS model follows:

Radiator $=\left(\mathrm{X}, \mathrm{Y}, \mathrm{S}, \delta_{\mathrm{ext}}, \delta_{\mathrm{int}}, \lambda\right.$, ta $)$

where

$\mathbf{X}: \mathrm{T}_{\max }, \mathrm{T}_{\mathrm{rd}}, \mathrm{T}_{\text {out }}, \Delta \mathrm{T}, \mathrm{P}_{\max }$, cost $_{\text {total }}$, cost $_{\text {rest_equipm }}$ (inputs).

S: transition states "Off", "On".

$\mathbf{Y}$ : $\mathrm{T}_{\text {room }}$ temperature provided for the room and pow $_{\text {cons }}$ the power consumed by the Radiator (outputs).

$\delta_{\text {ext }}$ : Calculates the temperature supplied to the room and the power consumed by the heater by the following formula :

$\left\{\begin{array}{l}\mathrm{T}_{\text {room }}=\mathrm{e}^{-t_{\max } / \tau}\left(\mathrm{T}_{\text {rd }}-\Delta \mathrm{T}-\mathrm{T}_{\text {out }}\right)+\mathrm{k}\left(1-\mathrm{e}^{-t_{\max } / \tau}\right) \mathrm{P}_{\max } \\ \text { pow }_{\text {cons }}=c o s \mathrm{t}_{\text {total }}-\cos \mathrm{t}_{\text {restequipm }} / c \text { ost } \mathrm{t}_{\text {generation/lw }}\end{array}\right.$

$\tau, \mathrm{k}, \operatorname{cost}_{\text {generation/lw }}:$ constants

$\delta_{\text {int }}: \delta_{\text {int }}=\varnothing$, the internal transition function, $\lambda: \lambda=\varnothing$, the output function and $\mathbf{t}_{\mathrm{a}}: \mathrm{t}_{\mathrm{a}}=\infty$, the advancement of time function.

\subsubsection{Representation of the sub-model Radiator using the JDEVS tool}

Figure 7 shows the Radiator sub-model modeled on JDEVS environmental:

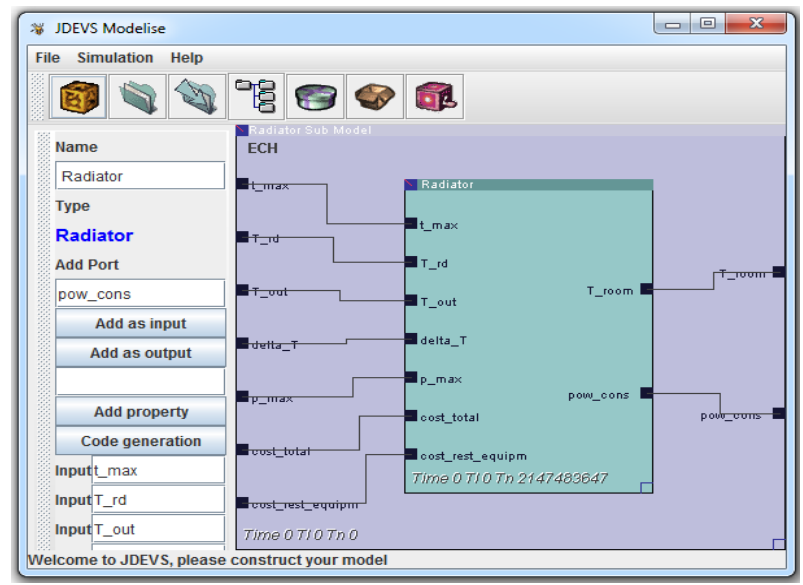

Fig 7: Representation of the Radiator model with JDEVS

After the simulation, the following results were obtained for power consumption:

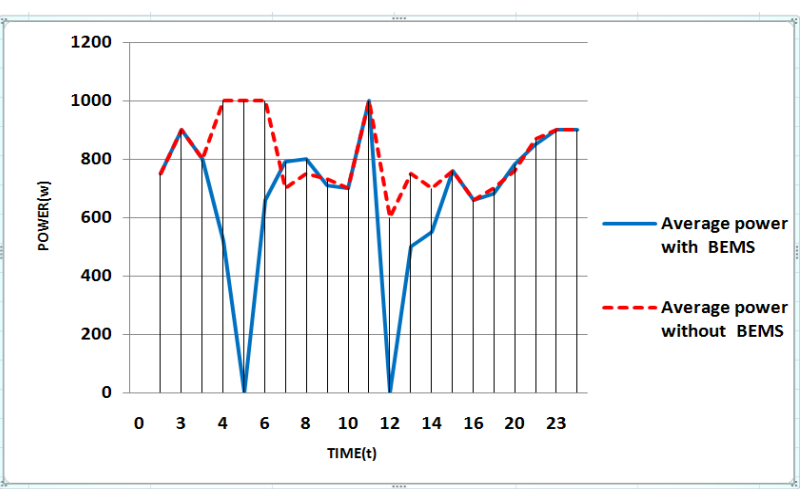

Fig 8: The average power consumed by the Radiator

\subsubsection{Discussion of results}

The figure 8 shows the behavior of the Radiator for 24h in the event of uncontrollable and controllable consumer consumption. In this figure, we can compare the average power consumed by the Radiator without reagent that consumed control when the reagent control is started, under the same operating conditions. From this curve, we find that the BEMS has not only smoothed the power not to exceed the purchase, but it allowed us to obtain an energy gain $\Delta \mathrm{P}$. The difference in area between the two curves is approximately $20 \%$.

That the optimizer receives measurement information from the HMI it will plan a minimum cost and transmit the resolver will give instructions to the controller which will also give commands to minimize cost to the Radiator and controls stop for some temporary equipment in peak hours. After these events, a reduction in energy consumption and satisfaction when necessary response is obtained. The energy manager has allowed doing economies for energy consumption by avoiding price spikes and overshoots subscription either by deletion or by shift operation temporary services during periods when there is less stress.

We compared two mechanisms: centralized proposed mechanism and distributed mechanism proposed by A. Shadi:

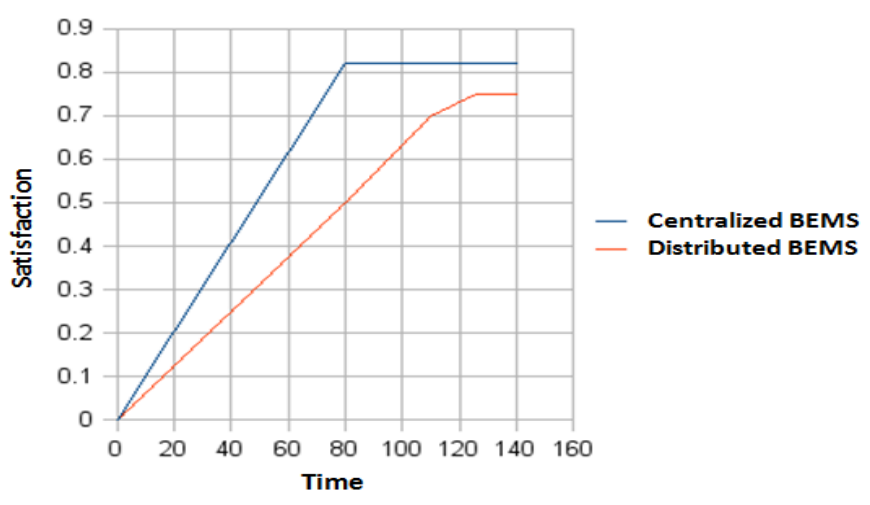

Fig 9: Satisfaction of solution with centralized and distributed system

In Figure 9, we note the quality of the solution obtained is $82 \%$ for the proposed centralized mechanism and $75 \%$ for the distributed mechanism. We also note that the research of the solution takes more time (35\% more) in the distributed mechanism compared with centralized. We note that the performances of centralized proposed system are better than those obtained with the distributed system. As against, level of robustness in the system based on distributed Multi-Agent Systems, is more robust than centralized system.

\section{CONCLUSION AND FUTURE PROSPECTS}

In this article we presented the modeling and the simulation of an energy management system of a multisource and a multiload building. Our contribution lies in the modeling and the simulation of this system with DEVS. We proposed a model that describes the studied system. Based on this model, we have developed an application using the tool of modeling and simulation JDEVS to simulate the behavior of the BEMS. The simulation of this BEMS gives good results for the reduction of energy consumption.

The continuation of this work will focus on several points. First, considering the implementation of the proposal work on more complex systems. For this, the future goal will be to 
model the previous BEMS with the formalism DEVS coupled with other tools of Soft Computing to touch all aspects of the real world.

\section{REFERENCES}

[1] Levermore, GJ., 1992. "An Application to Heating and Control, ”, E \& FN SPON . London, in Building Energy Management Systems.

[2] Kolokotsa, D., Niachou , K., Geros V., Kalaitzakis , K., Stavrakakis , GS., Santamouris, M., "Implementation of an integrated indoor environment and energy management system, " vol. 37 , no. 1, journal of Energy and Buildings, 2005, 93-99.

[3] Calvino, F., Gennusca , ML., Rizzo G., Scaccianoce, G., "The control of indoor thermal comfort requirements: introducing a fuzzy adaptive controller, " Vol. 36, No. 2, journal of Energy and Buildings, Feb.2004, 97-102.

[4] N. Morel, M. Bauer, M.,El-Khoury, J. Krauss, "Neurobat , a predictive and adaptive heating control system using artificial neural networks, "the international journal of Solar Energy, vol. 21 no. (2-3), 2000, 161-201.

[5] Paracha, ZJ., Doulai , P., 1998. "Load management : Technical and methods in electric power system, " vol.1 , in IEEE Energy Management and Power Delivery , 213217.

[6] K. Wacks, Utility load management using home automation. IEEE, the transaction journal of Consumer Electronics, vol. 37, issue. 2, 1991.

[7] Distributed Generation in Liberalised Electricity Markets by International energy agency , 2002. Available:http://gasunie.eldoc.ub.rug.nl/FILES/root/2002 13125958/3125958.pdf

[8] Negenborn, R., Houwing, M., De Schutter, B., Hellendoorn , H., 2008. "Adaptive prediction model accuracy in the control of residential energy resources" presented at the Proc. IEEE, the international conference of Control Application, 311-316.

[9] Henze, G. P., Dodier, R. H., 2002. "Adaptive optimal control of a grid -independent photovoltaic system", presented at the international conference of Solar Energy no. SED2002/1045, 2008,139-148.

[10] The Multisol ANR project PREBAJ Theme Days, Périchon, P., Ploix, $\quad$ S., 2010 Available:http://rp.urbanisme.equipement.gouv.fr/puca/a ctivites/prebat_310310/Ploix_Perrichon.pdf

[11] The REACTIVEHOME project. Available:http://multicom.imag.fr/spip.php?article153

[12] Ha , D. L., Ploix , S., Zamai, E., Jacomino , M., "Tabu search for the optimization of energy consumption houshold, " presented at Information Reuse and Integration, IEEE IRR, the international conference of Heuristic Systems Engineering, Waikoloa, Hawaii, USA, 2006, 86-92 .

[13] Ha , D. L., 2007. An advanced power management in the building to coordinate production and consumption system, Doctoral thesis, Dept. Electrical. Automation, Signal Processing . Univercity of Grenoble, France.

[14] Zeigler, B.P., 1976. Theory of Modelling and Simulation. Wiley \& Sons, New York.

[15] Capocchi, Laurent., 2005. Concurrent simulation of behavioral faults for discrete event systems : Application to digital circuits, Doctoral thesis, Univercity of pasquale paoli, Corsica.

[16] Shadi , A., 2009. Cooperation between board agents for managing energy in the habitat, Doctoral Thesis, Laboratory of Informatics of Grenoble - Imag Polytechnic Institute of Grenoble, France. 